\title{
Phonon assisted thermophoretic motion of gold nanoparticles inside carbon nanotubes
}

Schoen, Philipp A.E.; Walther, Jens Honore; Poulikakos, Dimos; Koumoutsakos, Petros

Published in:

Applied Physics Letters

Link to article, DOI:

10.1063/1.2748367

Publication date:

2007

Document Version

Publisher's PDF, also known as Version of record

Link back to DTU Orbit

Citation (APA):

Schoen, P. A. E., Walther, J. H., Poulikakos, D., \& Koumoutsakos, P. (2007). Phonon assisted thermophoretic motion of gold nanoparticles inside carbon nanotubes. Applied Physics Letters, 90(25), 253116.

https://doi.org/10.1063/1.2748367

\section{General rights}

Copyright and moral rights for the publications made accessible in the public portal are retained by the authors and/or other copyright owners and it is a condition of accessing publications that users recognise and abide by the legal requirements associated with these rights.

- Users may download and print one copy of any publication from the public portal for the purpose of private study or research.

- You may not further distribute the material or use it for any profit-making activity or commercial gain

- You may freely distribute the URL identifying the publication in the public portal 


\title{
Phonon assisted thermophoretic motion of gold nanoparticles inside carbon nanotubes
}

\author{
Philipp A. E. Schoen ${ }^{\text {a) }}$ \\ Laboratory for Thermodynamics in Emerging Technologies, ETH Zurich CH-8092, Switzerland \\ Jens $\mathrm{H}$. Walther ${ }^{\mathrm{b})}$ \\ Computational Science and Engineering Laboratory, ETH Zurich CH-8092, Switzerland and Department \\ of Mechanical Engineering, Technical University of Denmark, DK-2800 Kgs. Lyngby, Denmark \\ Dimos Poulikakos ${ }^{\mathrm{c})}$ \\ Laboratory for Thermodynamics in Emerging Technologies, ETH Zurich CH-8092, Switzerland \\ Petros Koumoutsakos ${ }^{\text {d) }}$ \\ Computational Science and Engineering Laboratory, ETH Zurich CH-8092, Switzerland
}

(Received 10 April 2007; accepted 21 May 2007; published online 22 June 2007; publisher error corrected 31 July 2007)

\begin{abstract}
The authors investigate the thermally driven mass transport of gold nanoparticles confined inside carbon nanotubes using molecular dynamics simulations. The observed thermophoretic motion of the gold nanoparticles correlates with the phonon dispersion exhibited by a standard carbon nanotube and, in particular, with the breathing mode of the tube. Additionally, the results show an increased static friction for gold nanoparticles confines inside a zig-zag carbon nanotube when increasing the size (length) of the nanoparticles. However, an unexpected, opposite trend is observed for the same nanoparticles inside armchair tubes. (C) 2007 American Institute of Physics.
\end{abstract}

[DOI: $10.1063 / 1.2748367]$

The atomically smooth surface and the rigidity of carbon nanotube (CNT) suggest an ideal medium for rapid nanoscale mass transport. Recent experimental and numerical studies have established a diffusion driven mass transport of light gases and liquids inside CNTs exceeding those of other nanoporous materials. ${ }^{1-6}$ Materials with high surface energy, such as metal nanoparticles physisorbed to multiwalled CNTs, can be transported across the CNT by applying a voltage gradient. ${ }^{7}$ The capability of filling CNTs with nanoparticles presents us with possibilities for the controlled motion and deposition of nanoparticles. ${ }^{8}$ A recent experimental application includes carbon nanotubes as "nanoextruders" for metal nanoparticles. ${ }^{9}$

In a previous work using molecular dynamics (MD) simulations we demonstrated CNT based nanoconveyors by showing thermophoretic, helical transport of nanoparticles confined inside carbon nanotubes. ${ }^{10}$ In this letter, we extend the study of thermophoresis and examine the influence of the coherent lattice vibrations on the nanotribological effects of the sliding gold nanoparticles confined inside CNTs.

The simulations consider an $(18,18)$ armchair and $(30,0)$ zig-zag CNT, both with a diameter $(d)$ of approximately $2.4 \mathrm{~nm}$ and length ranging between 20 and $100 \mathrm{~nm}$. The encapsulated gold nanoparticles contain $224\left(\mathrm{np}_{224}\right), 442$ $\left(\mathrm{np}_{442}\right), 924\left(\mathrm{np}_{924}\right)$, and $2274\left(\mathrm{np}_{2274}\right)$ gold atoms. The confined nanoparticles adopt a cylindrical shape with hemispherical caps (cf. inset Fig. 1). ${ }^{10}$ The cylindrical bodies have lengths ranging between approximately $1 \mathrm{~nm}\left(\mathrm{np}_{224}\right)$ and

\footnotetext{
${ }^{a)}$ Electronic mail: philipp.schoen@ethz.ch

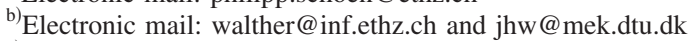

${ }^{c}$ Electronic mail: dimos.poulikakos@ethz.ch

${ }^{\mathrm{d})}$ Electronic mail: petros@ethz.ch
}

$12 \mathrm{~nm}\left(\mathrm{np}_{2274}\right)$. The resulting diameter of the nanoparticles is in all cases $1.75 \mathrm{~nm}$.

MD simulations are carried out using the FASTTUBE package $^{11}$ with the Glue ${ }^{12}$ model for gold. Details on the simulation procedure can be found in Refs. 10 and 13. A reduced tube is simulated additionally to a standard model CNT. The reduced tube is modeled as a system of uncorrelated harmonic oscillators, in which each carbon atom is connected to individual springs with spring constants

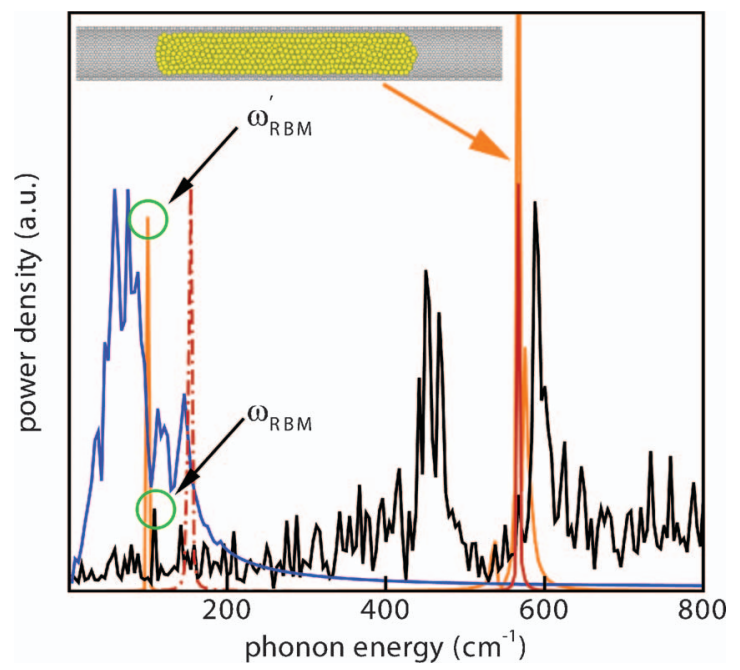

FIG. 1. (Color) Power DOS of a $\mathrm{np}_{2274}$ gold nanoparticle (blue) inside an artificial tube with $K_{10}=10000 \mathrm{~kJ} / \mathrm{mol} \mathrm{nm}^{2}$ (broken red line). The solid red line shows the artificial tube with $K_{130}=130000 \mathrm{~kJ} / \mathrm{mol} \mathrm{nm}{ }^{2}$. The $K_{130}$ model tube with imposed breathing mode is depicted as solid orange line. The DOS of a $(30,0)$ zig-zag CNT is added as well (black). The radial breathing mode of a CNT with $d \approx 2.4 \mathrm{~nm}$ is denoted by $\omega_{\mathrm{RBM}}$ $\approx 108.8 \mathrm{~cm}^{-1}$. That of the artificial tube appears as $\omega_{\mathrm{RBM}}^{\prime}$ Equilibrium temperature for all cases is $T=300 \mathrm{~K}$. (Inset) The artificial tube $\left(K_{130}\right)$. 
$K_{10}=10000 \mathrm{~kJ} / \mathrm{mol} \mathrm{nm}^{2}$ and $K_{130}=130000 \mathrm{~kJ} / \mathrm{mol} \mathrm{nm}{ }^{2}$, respectively.

We evaluate the nanotribological effects ${ }^{14,15}$ using three setups: first we evaluate the effect of correlated lattice vibrations, i.e., phonons, onto the thermophoretic mass transport by simulating an additional, reduced model CNT, and comparing it with a standard model tube, second we fix the position of the nanoparticle along the direction of the tube axis and measure the forces on the gold atoms resulting from the temperature gradient imposed along the axis of both the standard and the reduced CNT model. Since the reduced tube cannot conduct heat, we thermalize the system in $1 \mathrm{~nm}$ sections along the tube axis. In the last setup, we investigate the retarding friction force opposing the nanoparticle motion and measure the friction force when moving the nanoparticle through an isothermal standard CNT.

The phonon density of states (DOS) (cf. Fig. 1) of the reduced tubes has single frequencies corresponding to the eigenfrequencies of the harmonic oscillator: 154 and $567 \mathrm{~cm}^{-1}$ for the $K_{10}$ and $K_{130}$ models, respectively. The standard CNT model exhibits a continuous spectra including all eigenfrequencies of the CNT, and it is in good agreement with theoretical and experimental results. ${ }^{16,17}$ From the spectra (Fig. 1) we identify the radial breathing mode of $A_{1 g}$ symmetry at a frequency $\left(\omega_{\mathrm{RBM}}\right)$ of $108.8 \mathrm{~cm}^{-1}$, slightly exceeding the range of values $\left(95-105 \mathrm{~cm}^{-1}\right)$ determined experimentally. ${ }^{17}$ The measured frequencies $\left(65-130 \mathrm{~cm}^{-1}\right)$ for the gold nanoparticle are in good agreement with experimental results for bulk gold and gold nanoparticles. ${ }^{12}$

The phonon spectra of the $K_{10}$ tube overlap with the spectra of gold, cf. Fig. 1, and hence allows transmission of heat, ${ }^{18}$ as indicated by a noticeable heating of the encapsulated nanoparticle during the simulation. In contrast, no heat transfer occurs for the $K_{130}$ model, consistent with the disjoined phonon spectra (Fig. 1). We emphasize that, for both reduced models and for the imposed thermal gradients, no directed motion is observed for the nanoparticle, whereas the standard model CNT induced a thermophoretic motion similar to that observed in Ref. 10. We infer that the motion does not depend solely on the temperature gradient but also requires a coherent motion of the carbon atoms. In order to further assess this finding, we additionally impose onto the reduced $K_{130}$ tube a low energy radial breathing mode of $A_{1 g}$ symmetry. We model this breathing mode by imposing a radial oscillation on the mount point of the harmonic springs with a frequency of $\omega_{\mathrm{RBM}}^{\prime}=100 \mathrm{~cm}^{-1}$ (orange line Fig. 1) and with amplitudes in the range of $0.002-0.005 \mathrm{~nm}$. Only when imposing this additional breathing mode onto the reduced model tube, we observe a directed, thermophoretic motion of the gold nanoparticle similar to the motion observed in the standard CNT. The breathing mode of the CNT appears to reduce the frictional drag on the nanoparticle, and we attribute this effect to the release of the contact points between the two solid surfaces.

In order to examine the thermophoretic force exerted on the nanoparticle, we further fix the position of the nanoparticle on the center of the standard CNT. The resulting axial force response profile for the $\mathrm{np}_{2274}$ gold nanoparticle is shown in Fig. 2. The profile of the force distribution is asymmetric and integrates to a nonzero thermophoretic force. This effect becomes more pronounced for increasing temperature gradients. In addition, a radial force acts on the nanoparticle (inset of Fig. 2). This force is of higher magnitude at the hot Downloaded 18 Jun 2010 to 192.38.67.112. Redistribution subject

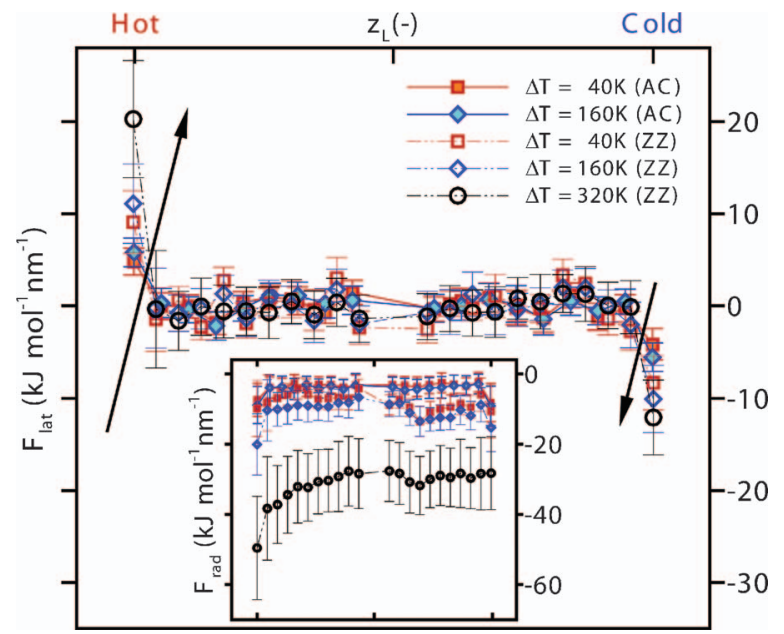

FIG. 2. (Color) Lateral $\left(F_{\text {lat }}\right)$ force response on the $\mathrm{np}_{2274}$ gold nanoparticle inside the $(18,18)$ armchair (AC) and $(30,0)$ zig-zag (ZZ) carbon nanotube. $z_{L}$ defines the dimensionless lengths of the nanoparticle. The arrows indicate increasing temperature differences along the CNT. (Inset) Radial force $\left(F_{\text {rad }}\right)$.

side and increases with increasing temperatures.

These forces trigger motion of the nanoparticle which involves an initial acceleration after which a constant, terminal velocity is reached when the retarding friction forces balance the thermophoretic driving force. In order to evaluate this relation, we measure the friction force $(F)$ per contact area $(A)$ retarding the motion of the nanoparticles sliding inside isothermal standard model CNTs with different chiral vectors, cf. Fig. 3.

The figure shows that the coefficient of dynamic friction, $\mu=F / A v$, where $v$ is the sliding velocity, displays an unexpected decrease with increasing size (length) when the $\mathrm{np}_{424}$ and $\mathrm{np}_{924}$ nanoparticles are sliding inside the armchair CNT. The opposite behavior is observed for the same nanoparticles

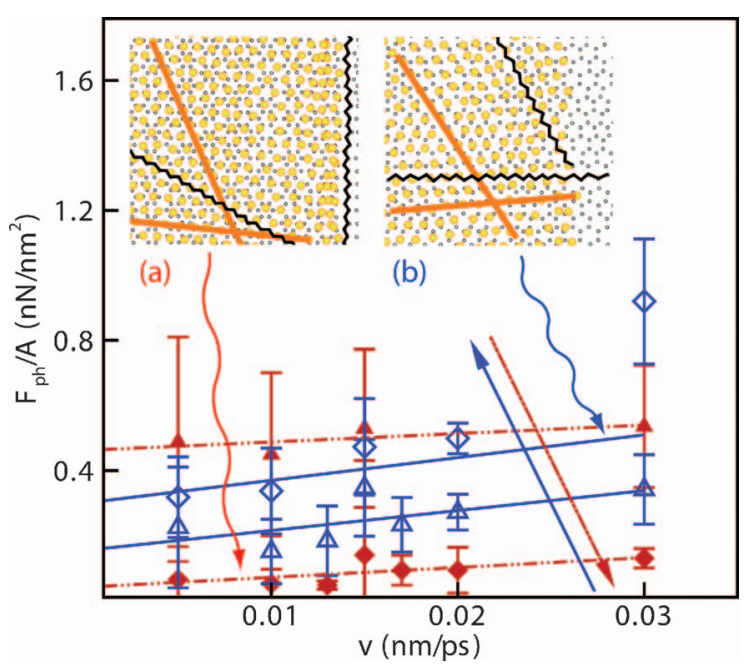

FIG. 3. (Color) Frictional forces on the $\mathrm{np}_{424}$ (triangle) and $\mathrm{np}_{924}$ (diamond) nanoparticles confined inside the armchair (red, filled symbols) and zig-zag (blue, open symbols) CNT versus drift velocity $(v)$. The considered velocities correspond to values measured when driving these nanoparticles by temperature gradients between 0.4 and $2 \mathrm{~K} / \mathrm{nm}$. Two-dimensional unfolded atomic structure of the first gold layer nanotube $\left(\mathrm{np}_{2274}\right)$ covering the unfolded graphene sheet of the a) armchair and (b) zig-zag CNTs. The red lines indicate the angle of alignment of the gold atoms and the black lines the zig-zag orientation of carbon atoms in the graphene layer: (a) $30^{\circ}$ and $90^{\circ}$ for the armchair and (b) $0^{\circ}$ and $60^{\circ}$ for the zig-zag. 
when confined inside the comparable $(30,0)$ zig-zag standard model CNT (Fig. 3). In both cases, i.e., the $\mathrm{np}_{424}$ and $\mathrm{np}_{924}$ gold nanoparticle sliding inside the i) armchair and ii) zigzag CNT, the coefficient of dynamic friction is found to be independent of the translational velocity and similar for the different carbon nanotubes (see slope of the fits in Fig. 3). Nanoparticles sliding inside longer CNTs $(\approx 100 \mathrm{~nm}$ length) confirm the findings of very different friction forces when confined inside armchair and zig-zag CNTs, respectively. ${ }^{10}$

These results suggest that the differences in the static friction force $(v \rightarrow 0)$ for various combinations of chiral vectors and larger nanoparticles, e.g., $\mathrm{np}_{924}$, are associated with the different levels of commensuration at the gold-carbon interface. In order to further elucidate this assessment, we present the unfolded cylindrical stacked gold nanoparticles ${ }^{10,19}$ and the CNTs to obtain two-dimensional sheets of gold on carbon atoms [see Figs. 3(a) and 3(b)]. We note that the angle of alignment of gold atoms almost coincides with the zig-zag patterned carbon atoms of the unfolded zig-zag CNT, denoting a high degree of commensuration [Fig. 3(b)]. However, the same does not hold for the unfolded nanoparticle confined inside the armchair CNT showing no coincidence of the aligned gold atoms with the zig-zag patterned carbon atoms [Fig. 3(a)]. These results suggest that the reduced degree of commensuration decreases the static friction of gold nanoparticles confined inside armchair CNTs when compared with particles confined inside zig-zag CNTs (Fig. 3).

The opposite holds when comparing the friction of small nanoparticles $\left(\mathrm{np}_{424}\right)$ confined inside the armchair and zigzag CNT, respectively (Fig. 3). In the case of the $\mathrm{np}_{424}$, the static friction is significantly smaller inside the zig-zag CNT than inside the armchair CNT. We believe that the length of the small gold nanoparticles inside the CNTs is too short to "feel" the far distance symmetry of the armchair CNT having straight zig-zag lines parallel with the tube axis [Fig. 3(a)]. Small gold nanoparticles moving through the armchair CNT follow the zig-zig lines with angle $30^{\circ}$, as shown in Ref. 10 . This motion is associated with a higher friction of small nanoparticles sliding inside an armchair CNT when compared with a small nanoparticle sliding inside a zig-zag tube having zig-zag lines with $60^{\circ}$ orientation (Fig. 3). These findings accentuate a predominant effect of surface corrugation on diffusion and sliding of solid nanoclusters. ${ }^{14,20,21}$

In summary, we have shown that nanoparticles move only under the assistance of the breathing mode of the CNT, which is responsible for the release of gold-carbon contact points. We observe an unexpected decrease of the static frictional drag for increasing nanoparticle sizes when confined inside armchair CNTs.

This work was supported by the Swiss National Science Foundation under Grant No. 2000-067738/1. One of the authors (J.H.W.) acknowledges additional support by the Julie Damm's Studiefond.

${ }^{1}$ H. Chen, J. K. Johnson, and D. S. Sholl, J. Phys. Chem. B 110, 1971 (2006).

${ }^{2}$ A. I. Skoulidas, D. S. Sholl, and J. K. Johnson, J. Chem. Phys. 124, 054708 (2006)

${ }^{3}$ J. K. Holt, H. G. Park, Y. Wang, M. Stadermann, A. B. Artyukhin, C. P. Grigoropoulos, A. Noy, and O. Bakajin, Science 312, 1034 (2006).

${ }^{4}$ U. P. G. Gonnet Zimmerli and J. H. Walther, Nano Lett. 5, 1017 (2005).

${ }^{5}$ M. Majumder, N. Chopra, R. Andrews, and B. J. Hinds, Nature (London) 438, 44 (2005).

${ }^{6}$ A. I. Skoulidas, D. M. Ackerman, J. K. Johnson, and D. S. Sholl, Phys. Rev. Lett. 89, 185901 (2002).

${ }^{7}$ B. C. Regan, S. Aloni, R. O. Ritchie, U. Dahmen, and A. Zettl, Nature (London) 428, 924 (2004).

${ }^{8}$ P. M. Ajayan and S. Iijima, Nature (London) 361, 333 (1993).

${ }^{9}$ L. Sun, F. Banhart, A. V. Krasheninnikov, J. A. Rodriguez-Manzo, M. Terrones, and P. M. Ajayan, Science 312, 1199 (2006).

${ }^{10}$ P. A. E. Schoen, J. H. Walther, S. Arcidiacono, D. Poulikakos, and P. Koumoutsakos, Nano Lett. 6, 1910 (2006).

${ }^{11}$ J. H. Walther, R. Jaffe, T. Halicioglu, and P. Koumoutsakos, J. Phys. Chem. B 105, 9980 (2001).

${ }^{12}$ F. Ercolessi, M. Parrinello, and E. Tosatti, Philos. Mag. A 58, 213 (1988).

${ }^{13}$ E. M. Kotsalis, J. H. Walther, and P. Koumoutsakos, Int. J. Multiphase Flow 30, 995 (2005).

${ }^{14}$ J. C. Hamilton, Phys. Rev. Lett. 77, 885 (1996).

${ }^{15}$ W. D. Luedtke and U. Landman, Phys. Rev. Lett. 82, 3835 (1999).

${ }^{16}$ R. Saito, T. Takeya, and T. Kimura, Phys. Rev. B 57, 4145 (1998).

${ }^{17}$ S. Reich, C. Thomsen, and J. Maultzsch, Carbon Nanotubes-Basic Concepts and Physical Properties (Wiley, Berlin, 2004), 1, p. 141.

${ }^{18}$ B. Li, L. Wang, and G. Casati, Phys. Rev. Lett. 93, 184301 (2004).

${ }^{19}$ S. Arcidiacono, J. H. Walther, D. Poulikakos, D. Passerone, and P. Koukoumtsakos, Phys. Rev. Lett. 94, 105502 (2005).

${ }^{20}$ D. S. Sholl and K. A. Fichthorn, Phys. Rev. Lett. 79, 3569 (1997).

${ }^{21}$ D. D. Sholl and R. T. Skodje, Phys. Rev. Lett. 75, 3158 (1995). 